\title{
La météorologie de Jupiter
}

\author{
Thierry Fouchet \\ Laboratoire d'études spatiales et d'instrumentation en astrophysique \\ Observatoire de Paris \& Université Pierre-et-Marie-Curie \\ 5, place Jules-Janssen - 92195 Meudon Cedex \\ thierry.fouchet@obspm.fr
}

\section{Résumé}

Jupiter, la plus grande et la plus massive des planètes du système solaire, est une planète-atmosphère : le gaz constitue la quasi-totalité de sa masse et de son volume. Cette planète est un laboratoire grandiose pour la dynamique des fluides, pour la chimie atmosphérique, pour comprendre l'histoire du système solaire. On pourrait en apprendre beaucoup sur notre propre atmosphère, mais on ne comprend encore que très peu de choses. Quelle est la source des puissants vents zonaux de Jupiter ? Quels sont l'origine et le destin de ses tourbillons ? Quel agent colore ses nuages ? Voici une synthèse de ce que l'on sait et de ce que l'on ignore sur l'atmosphère de Jupiter.

\section{Abstract}

\section{The meteorology of Jupiter}

Jupiter, the largest and the most massive of the solar system's planets, is a planet-atmosphere: almost the whole of its mass and volume is gas. This planet is a wonderful laboratory for fluid dynamics, atmospheric chemistry, and the understanding of solar system history. We could learn a lot about our own atmosphere from Jupiter, but we still lack basic understanding. What drives the powerful zonal winds of Jupiter? What are the origin and destiny of its vortices? What colours its clouds? Here is a global picture of what is known and unknown about Jupiter's atmosphere. upiter est la plus massive et la plus imposante des planètes du système solaire. Sa masse approche 320 fois la masse terrestre et son rayon équivaut à douze rayons terrestres. Mais Jupiter se distingue également par une densité très inférieure à celle de la Terre $-1,3 \mathrm{~g} / \mathrm{cm}^{3}$ contre $5,3 \mathrm{~g} / \mathrm{cm}^{3}-$, ce qui ne peut s'expliquer que par une composition chimique radicalement différente : si la Terre est principalement constituée de roches (silicates) et de métaux, Jupiter est avant tout composée de gaz, beaucoup plus légers. On peut donc considérer Jupiter comme une immense atmosphère, sans topographie ni océan. Nul doute que cette particularité induit des différences majeures entre la météorologie jovienne et la météorologie terrestre.

D'autres paramètres jouent également un rôle dans la météorologie jovienne, tel le flux solaire qui, à la distance héliocentrique de Jupiter, n'atteint que $3,3 \%$ du flux solaire reçu par la Terre. Ou encore l'existence d'une source d'énergie interne qui représente $70 \%$ de l'énergie solaire absorbée par la planète : en effet, le cœur de Jupiter n'évacue que lentement l'énergie gravitationnelle concentrée lors de la

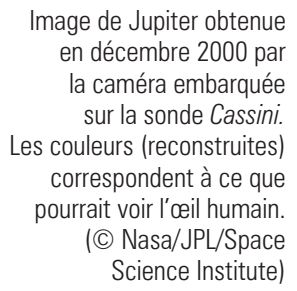
en décembre 2000 par la caméra embarquée sur la sonde Cassini. Les couleurs (reconstruites) correspondent à ce que pourrait voir l'œil humain. (C) Nasa/JPL/Space Science Institute)

formation de la planète et de l'énergie, sous forme de chaleur, est encore évacuée vers l'extérieur par convection.

\section{L'étude de Jupiter}

L'étude de la planète Jupiter - et de sa météorologie en particulier - repose presque exclusivement sur la télédétection, que ce soit par des observations astronomiques classiques à l'aide de télescopes au sol ou en orbite terrestre ou, plus récemment, par des missions spatiales dédiées (voir encadré page 20). Une seule mission spatiale, la mission Galileo de la Nasa, a permis d'obtenir des mesures in situ de la composition 


\section{L'exploration spatiale de Jupiter}

Les premières sondes spatiales à atteindre la planète Jupiter furent les sondes Pioneer 10 et Pioneer 11 dans les années 1970. Puis, en 1979 et 1980, les deux sondes Voyager 1 et Voyager 2 survolèrent la planète avant de poursuivre leur voyage vers les confins du système solaire. Ces deux sondes ont révolutionné notre connaissance de l'atmosphère jovienne en cartographiant pour la première fois de manière précise ses vents et ses champs de température, d'humidité et de composition chimique. De 1995 à 2001, la mission Galileo fut un grand succès, et cela à double titre. D'une part, la sonde de descente mesura pour la première fois in situ la température, la composition chimique et les vents joviens. De l'autre, cette sonde fut la première à se mettre en orbite autour d'une planète géante, Jupiter en l'occurrence. À bord de cet orbiteur, la caméra a fourni, avec une résolution spatiale sans précédent, des images des nuages de Jupiter et de la surface des principaux satellites joviens. Enfin, en 2000-2001, la mission Cassini a survolé Jupiter au cours de son voyage vers Saturne. Près de vingt ans après les missions Voyager, des instruments semblables, mais de tech-

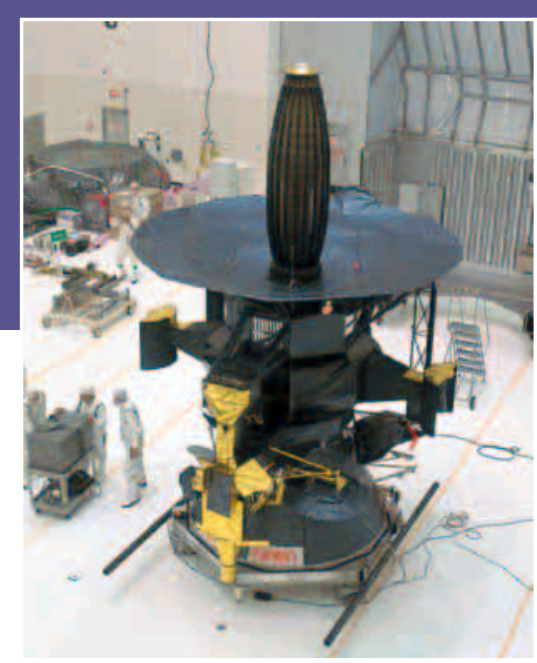

chimique, de la température, de la structure nuageuse et des vents de l'atmosphère jovienne. Si cette mission a fourni des résultats importants, son caractère éphémère (une heure de mesures) et local n'a pas diminué l'intérêt de la télédétection qui apporte une vision plus globale et plus continue dans le temps de la planète.

Trois techniques de télédétection sont utilisées :

- La spectroscopie, de l'ultraviolet aux ondes millimétriques, sert à déterminer et à quantifier la composition chimique des gaz atmosphériques et des aérosols, mais également à sonder la température de l'atmosphère dans l'infrarouge thermique.

- L'imagerie permet d'observer la structure nuageuse de la planète (altitude et nologie beaucoup plus moderne, ont scruté les quelques changements intervenus dans la météorologie remarquablement stable de Jupiter.

Préparation de Galileo

dans les laboratoires de la Nasa. (C Nasa)

épaisseur des nuages) et de suivre son évolution temporelle afin de mesurer la vitesse des vents.

- La technique de l'occultation donne accès au profil vertical de la température et, éventuellement, de la composition chimique. Lorsque la planète passe devant une étoile ou une sonde, une fraction de la lumière de l'étoile ou de la sonde est réfractée dans l'atmosphère de la planète avant d'atteindre l'observateur.

Ces trois techniques peuvent être mises en œuvre indifféremment sur des télescopes ou à bord de missions spatiales. En général, les missions spatiales donnent accès à une haute résolution à la surface de la planète et à des gammes de fréquence inaccessibles à travers l'atmosphère terrestre; de plus, elles observent la planète de manière quasi c on ti n u e durant plusieurs mois ou

Figure 1 - Profil de température de Jupiter en fonction de la pression, de $10^{-6} \mathrm{hPa}$ jusqu'à $10^{10} \mathrm{hPa}$. L'atmosphère est découpée en deux régions

la stratosphère où la température croît avec l'altitude et la troposphère où la température décroît avec l'altitude. La limite entre les deux régions se situe vers $100 \mathrm{hPa}$. La couverture nuageuse de Jupiter se situe entre 5000 et $500 \mathrm{hPa}$. Plus profondément dans la planète, il doit exister d'autres régions de condensation, mais que le qualificatif de nuages ne décrit pas forcément bien. Les métaux condensent entre 1000 et $2000 \mathrm{~K}$. Plus en profondeur, l'hydrogène subit une transition de phase depuis l'hydrogène moléculaire vers l'hydrogène métallique.

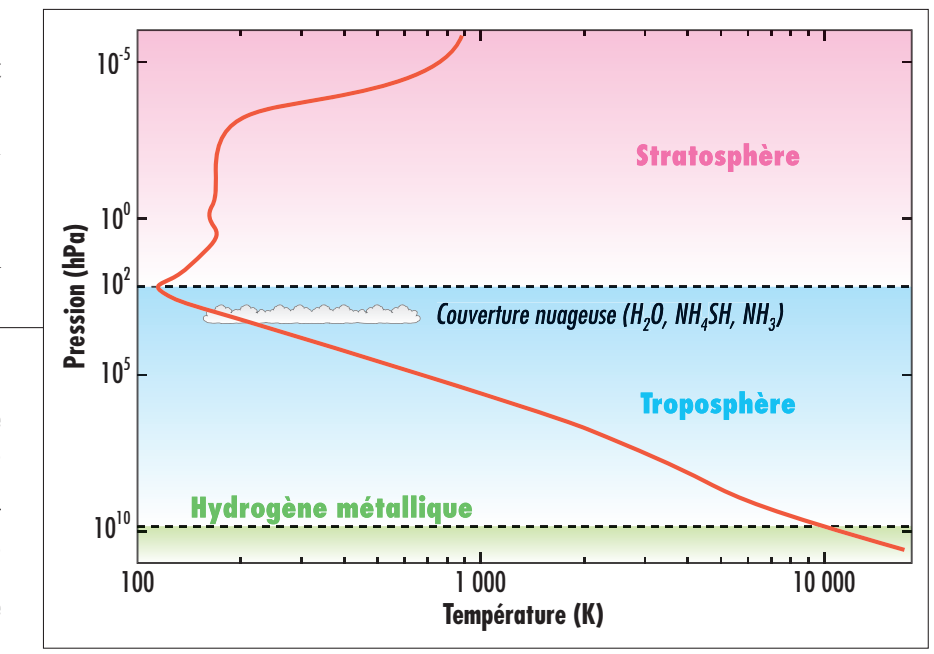

plusieurs années. De leur côté, les télescopes, qui servent à toute la communauté astronomique, ne peuvent consacrer que quelques nuits par an à l'observation de Jupiter. En revanche, leurs instruments sont plus sensibles que les instruments spatiaux. Ces derniers sont en effet réalisés à l'aide de technologies plus frustes, capables de résister à un long trajet interplanétaire, et qui finalement datent de plusieurs années à leur arrivée dans la « banlieue » de Jupiter. Ces différents avantages et inconvénients font que missions spatiales et observations astronomiques classiques coexistent toujours actuellement.

Il faut de plus se rendre compte que ces méthodes de télédétection ne font qu'effleurer le géant Jupiter. En effet, la télédétection est globalement limitée par la couche nuageuse ; les fenêtres existantes dans les fréquences radio permettent seulement de sonder 
l'atmosphère jusqu'à quelques dizaines de milliers d'hectopascals. Ainsi, on n'a accès directement qu'à la stratosphère (région où la température croît avec l'altitude) et à la limite supérieure de la troposphère (région où la température décroît avec l'altitude) de Jupiter. Tout l'intérieur de la planète demeure invisible. Il n'est accessible que via des modèles numériques qui reproduisent le flux d'énergie interne évacué par la planète, le champ magnétique et le champ de gravité mesurés par les sondes interplanétaires. On sait ainsi que l'intérieur de Jupiter est chaud, ce qui assure qu'il demeure fluide. Dans ces conditions, l'hydrogène devient métallique vers $8.10^{\circ} \mathrm{hPa}$. En revanche, on ignore s'il existe un cœur solide au centre de la planète. La figure 1 résume la structure globale de l'atmosphère de Jupiter.

\section{Apparence de Jupiter, couches nuageuses et vents}

\section{Zones et ceintures}

Depuis les premiers observateurs jusqu'à nos jours, l'apparence de Jupiter n'a que très peu changé. Elle est le reflet de la couverture nuageuse située à des pressions comprises entre 500 et $1500 \mathrm{hPa}$. Elle présente une remarquable structure en bandes alternativement brillantes et sombres, structure symétrique par rapport à l'équateur (figure $2)$. Traditionnellement, les bandes brillantes sont appelées zones et les bandes sombres ceintures. Les zones sont des régions où la couverture nuageuse est plus dense, plus uniforme et plus élevée que dans les ceintures. La lumière solaire y est plus efficacement réfléchie, si bien que les zones apparaissent brillantes par rapport aux ceintures. Si cette structure est globalement stable depuis des siècles, les frontières entre zones et ceintures fluctuent quelque peu en latitude. La couleur de ces bandes évolue également au cours du temps.

\section{Les vents}

Les images à haute résolution spatiale montrent que la texture et la couleur des nuages varient à l'échelle de quelques kilomètres. À cette résolution, le suivi des nuages permet de mesurer

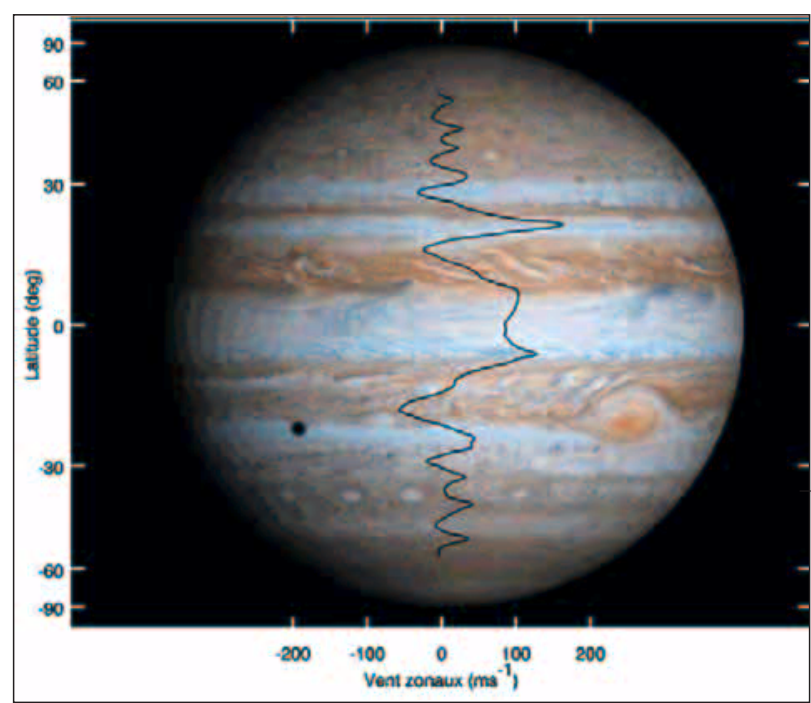

Figure 2 - Image de Jupiter obtenue par la sonde Cassini. Le profil des vents zonaux est superposé à l'image. On peut voir quelques structures célèbres de la planète : la grande tache rouge dans le quart sud-est, des ovales blancs dans le quart sud-ouest. La tache sombre dans I'hémisphère sud est l'ombre du satellite Europe sur la planète. (C) Nasa/ Cassini ; d'après Limaye (1986) pour le profil de vent)

la vitesse des vents avec une précision de l'ordre de quelques mètres par seconde. Sur les planètes telluriques ${ }^{(1)}$, la vitesse des vents est exprimée dans le référentiel en rotation de la planète, habituellement confondu avec sa surface solide. Sur les planètes géantes comme Jupiter, en l'absence d'une telle surface, la période de rotation choisie est celle des ondes radio kilométriques émises par la planète ( 9 h 55 min 29,71 s), qui est en principe liée à la rotation de l'intérieur de la planète via le champ magnétique. La mesure des vents montre une alternance entre des courants-jets soufflant d'ouest en est, qui peuvent être extrêmement violents (jusqu'à environ $150 \mathrm{~m} / \mathrm{s}$ ), et des courants-jets soufflant d'est en ouest, un peu moins puissants (jusqu'à environ $50 \mathrm{~m} / \mathrm{s}$ )

Cette structure dynamique est étroitement liée à la structure en bandes de Jupiter. En effet, les courants-jets d'ouest sont situés à la frontière de latitude supérieure des zones et à la frontière de latitude inférieure des ceintures, tandis que les courants-jets d'est sont situés à la frontière de latitude inférieure des zones et à la frontière de latitude supérieure des ceintures. Ainsi, les zones présentent une rotation globale dans le sens des aiguilles d'une montre dans l'hémisphère nord et dans le sens inverse dans l'hémisphère sud ; ce sont des anticyclones. Les ceintures présentent un sens de rotation opposé à celui des zones ; ce sont des régions dépressionnaires.

\section{Les tourbillons}

Il existe de nombreuses structures tourbillonnaires à l'intérieur des zones et des ceintures, dont la plus connue est la existent, tels les ova-

grande tache rouge. D'autres structures tourbillonnaires les blancs vers $33^{\circ} \mathrm{S}$ ou d'autres ovales plus petits vers $41^{\circ} \mathrm{S}, 34^{\circ} \mathrm{N}, 40^{\circ} \mathrm{N}$ et $45^{\circ} \mathrm{N}$. Ces tourbillons tendent à avoir le même sens de rotation que la bande dans laquelle ils sont immergés : les anticyclones sont situés dans les zones, les dépressions dans les ceintures. Ainsi, la grande tache rouge est un gigantesque anticyclone situé dans la zone équatoriale sud (figure 2). Comme tous les tourbillons anticycloniques joviens, la tache est elliptique avec un grand axe aligné dans la direction est-ouest. Son grand axe actuel mesure $17000 \mathrm{~km}$ et son petit axe $12500 \mathrm{~km}$. Au centre de la tache, la vitesse des vents est très faible, tandis qu'à l'extérieur on observe une vitesse moyenne de $120 \mathrm{~m} / \mathrm{s}$ avec des pointes à $190 \mathrm{~m} / \mathrm{s}$. Toutefois, l'ellipticité de la grande tache rouge tend à diminuer : en 1880 , son grand axe atteignait $39000 \mathrm{~km}$. Les structures cycloniques joviennes sont plus difficiles à observer car moins contrastées, mais bien présentes. Leur structure est plus chaotique, parfois sous la forme de filaments.

En règle générale, plus les tourbillons sont petits, plus leur durée de vie est courte. Ainsi, le plus grand des tourbillons joviens, la grande tache rouge, est observé clairement depuis 1831 , mais semble présent dans les dessins de Cassini dès 1665. La disparition des tourbillons se produit, soit par un cisaillement dans une région de fort gradient de vent, soit par coalescence entre tourbillons : la grande tache rouge

(1) Les planètes telluriques sont les planètes dans lesquelles roches (silicates) et métaux constituent la majeure partie de la masse. Dans le système solaire, ce sont Mercure, Vénus, la Terre et Mars. Jupiter et Saturne sont qualifiées de géantes gazeuses (majeure partie de la masse sous forme de gaz) et Uranus et Neptune de géantes glacées (majeure partie de la masse sous forme de glace). 
avale souvent des petits anticyclones qui passent à proximité. Récemment, on a assisté au regroupement des trois ovales situés à $33^{\circ} \mathrm{S}$. Présents depuis 1940 , de petit et grand axe d'environ 5000 et $10000 \mathrm{~km}$, ils étaient régulièrement espacés en longitude. À la fin des années 1990, l'un des ovales a ralenti sa course et les deux autres l'ont rattrapé pour former un train de tourbillons. Puis, en 1998, deux ovales ont fusionné. Deux ans plus tard, les deux derniers ovales ont également fusionné (figure 3).

\section{Composition chimique de l'atmosphère et des nuages}

La composition chimique d'une atmosphère joue un rôle essentiel dans sa dynamique et sa météorologie :

- Elle conditionne la densité du gaz et son échelle de hauteur, qui quantifie la décroissance de la pression avec l'altitude ; l'échelle de hauteur est de $25 \mathrm{~km}$ sur Jupiter.

- Elle influe sur le champ de température de la planète, via la quantité d'énergie solaire absorbée et la quantité de rayonnement thermique émise, mais aussi via les régions (latitude, longitude et altitude) où ces rayonnements sont absorbés et émis.

- Enfin, la composition chimique, l'altitude et l'épaisseur des nuages dépendent de l'abondance des molécules susceptibles de condenser.

Déterminer précisément quels gaz composent l'atmosphère jovienne et en quelles quantités constitue donc l'un des axes principaux de la recherche actuelle : il manque toujours des mesures précises sur des gaz d'importance météorologique majeure.

\section{Les principaux gaz}

L'atmosphère de Jupiter est essentiellement constituée d'hydrogène moléculaire $\left(\mathrm{H}_{2}\right)$ et d'hélium $(\mathrm{He})$, respectivement pour $86 \%$ et $14 \%$ de la fraction volumique. $\mathrm{H}_{2}$ est détecté par spectroscopie dans l'infrarouge. L'hélium n'est pas actif, mais sa présence et sa quantité affectent la forme et l'intensité des émissions de $\mathrm{H}_{2}$. Il est donc détecté indi-

Tableau 1 - Abondance relative des gaz dans l'atmosphère de Jupiter. (D’après Taylor et al., 2004)

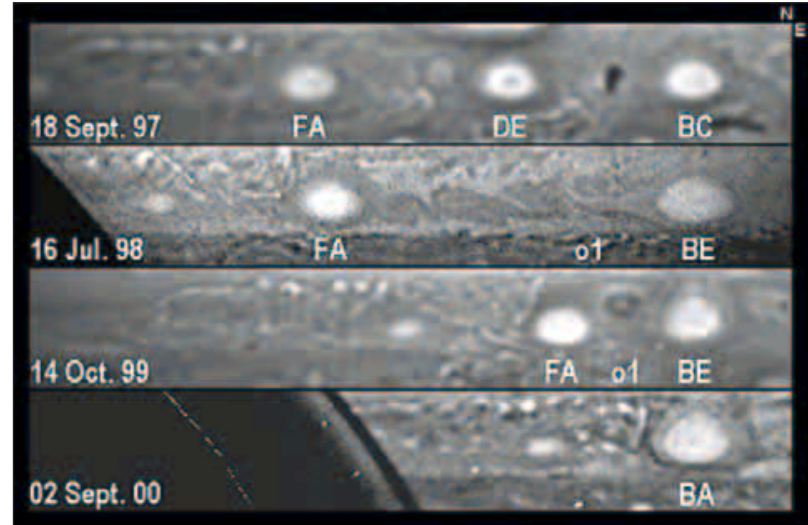

\section{Autres composés carbonés}

Plus à l'intérieur de la planète, lorsque la température et la pression dépassent $400 \mathrm{~K}$ et quelques millions de pascals, la thermodynamique impose d'autres molécules stables. Dans ces conditions, le carbone doit exister sous

Figure 3 - Ces quatre images dues au télescope spatial Hubble montrent les différentes étapes de la coalescence des ovales blancs situés dans l'hémisphère sud de Jupiter. Les ovales sont des tourbillons anticycloniques dont le grand axe est compris entre 8000 et $12000 \mathrm{~km}$. Entre les ovales blancs, les images montrent des structures sombres peu contrastées : ce sont des tourbillons dépressionnaires. En septembre 1997, trois ovales (FA, DE et $B C$ ) sont présents, régulièrement espacés. En juillet 1998, les ovales DE et $B C$ ont fusionné en un seul (BE). Entre juillet 1998 et octobre 1999, la distance entre les ovales FA et $\mathrm{BE}$ a rapidement diminué et, en septembre 2000, ils ont fusionné en un seul ovale (BA). Malheureusement, au moment des fusions, Jupiter n'était pas observable depuis le télescope spatial Hubble. Le nord est situé en haut de l'image, l'est vers la droite. (@ Nasa/JPL/WFPC2)

rectement. Toutefois, les mesures les plus précises de leur fraction volumique ont été obtenues in situ par le spectromètre de masse de la sonde de descente Galileo. Dans un tel environnement hydrogéné, aux conditions de température et de pression de la partie observable de l'atmosphère, les principales molécules ne peuvent être que des molécules réduites : un atome donné formera une molécule en complétant toutes ses liaisons avec un atome d'hydrogène. Ainsi, les principaux gaz traces sont l'eau $\left(\mathrm{H}_{2} \mathrm{O}\right)$, le méthane $\left(\mathrm{CH}_{4}\right)$ et l'ammoniac $\left(\mathrm{NH}_{3}\right)$, puis viennent le sulfure d'hydrogène $\left(\mathrm{H}_{2} \mathrm{~S}\right)$, la phosphine $\left(\mathrm{PH}_{3}\right)$, l'arsine $\left(\mathrm{AsH}_{3}\right)$ et le germane $\left(\mathrm{GeH}_{4}\right)$. Le spectromètre de masse de la sonde Galileo a également détecté les gaz rares argon, xénon et krypton ( $\mathrm{Ar}, \mathrm{Xe}, \mathrm{Kr}$ ), inobservables par spectroscopie en télédétection. Le tableau 1 résume la composition chimique de l'atmosphère jovienne. forme de monoxyde de carbone (CO) plutôt que sous forme de méthane. Lorsque, par convection ou par turbulence, une parcelle de gaz est transportée vers l'extérieur de l'atmosphère, elle se détend, se refroidit et le CO s'y transforme en $\mathrm{CH}_{4}$ (via la réaction $\mathrm{CO}+3 \mathrm{H}_{2} \leftrightarrow \mathrm{CH}_{4}+\mathrm{H}_{2} \mathrm{O}$ ). Toutefois, cette conversion n'est pas instantanée, mais obéit à une cinétique : il existe une compétition entre le temps mis par la parcelle pour atteindre les couches observables et le temps mis par le $\mathrm{CO}$ pour se convertir en $\mathrm{CH}_{4}$. Les observations montrent que la réaction est plus lente que le mélange vertical : la masse d'air monte, se refroidit et atteint les couches atmosphériques observables avant que tout le $\mathrm{CO}$ ne se soit converti en méthane. En effet, la quantité de CO mesurée vers $1000 \mathrm{hPa}$, 1 partie par milliard, ne correspond pas à ce qui est attendu à l'équilibre thermodynamique. Cet écart à l'équilibre renseigne sur la dynamique et la chimie des couches situées sous les nuages.

Il existe également des molécules provenant de la photochimie initiée par le rayonnement ultraviolet solaire. Celui-ci

\begin{tabular}{l|l|l} 
Molécule & Fraction volumique & Technique de mesure \\
\hline $\mathrm{H}_{2}$ & $86,2 \%$ & In situ \\
\hline $\mathrm{He}$ & $13,6 \%$ & In situ \\
\hline $\mathrm{CH}_{4}$ & $0,2 \%$ & Télédétection et in situ \\
\hline $\mathrm{H}_{2} \mathrm{O}$ & $>5.10^{-4}$ & In situ \\
\hline $\mathrm{NH}_{3}$ & $6,6.10^{-4}$ & In situ \\
\hline $\mathrm{H}_{2} \mathrm{~S}$ & $9.10^{-5}$ & In situ \\
\hline $\mathrm{PH}_{3}$ & $6.10^{-7}$ & Télédétection \\
\hline $\mathrm{GeH}_{4}$ & $7.10^{-10}$ & Télédétection \\
\hline $\mathrm{AsH}_{3}$ & $3.10^{-10}$ & Télédétection \\
\hline $\mathrm{C}_{2} \mathrm{H}_{6}, \mathrm{C}_{2} \mathrm{H}_{4}, \mathrm{C}_{2} \mathrm{H}_{2 \prime}$ & $\begin{array}{l}\text { Variable. Présents } \\
\mathrm{CH}_{3} \mathrm{C}_{2} \mathrm{H}, \mathrm{C}_{4} \mathrm{H}_{2}, \mathrm{C}_{6} \mathrm{H}_{6}\end{array}$ & $\begin{array}{l}\text { essentiellement dans } \\
\text { la stratosphère. }\end{array}$ \\
& & Télédétection
\end{tabular}




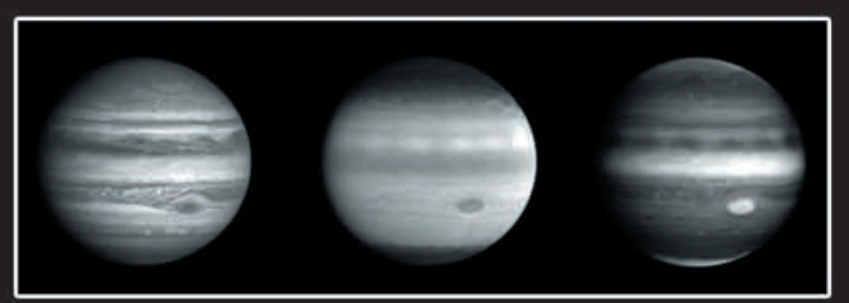

Images de Jupiter obtenues dans le bleu (à gauche), l'ultraviolet (au centre) et le proche infrarouge (à droite). Dans le bleu, Jupiter est semblable à ce que l'on pourrait voir à l'œil nu et ses contrastes sont dus aux nuages troposphériques.

Dans l'ultraviolet et le proche infrarouge, l'image révèle

les aérosols dans la stratosphère. Dans l'ultraviolet, les aérosols apparaissent sombres (plus sombres que la diffusion Rayleigh du gaz). Dans l'infrarouge, ils apparaissent brillants car l'infrarouge proche est absorbé par le méthane stratosphérique. (C STScl/Aura/Nasa/ESA)
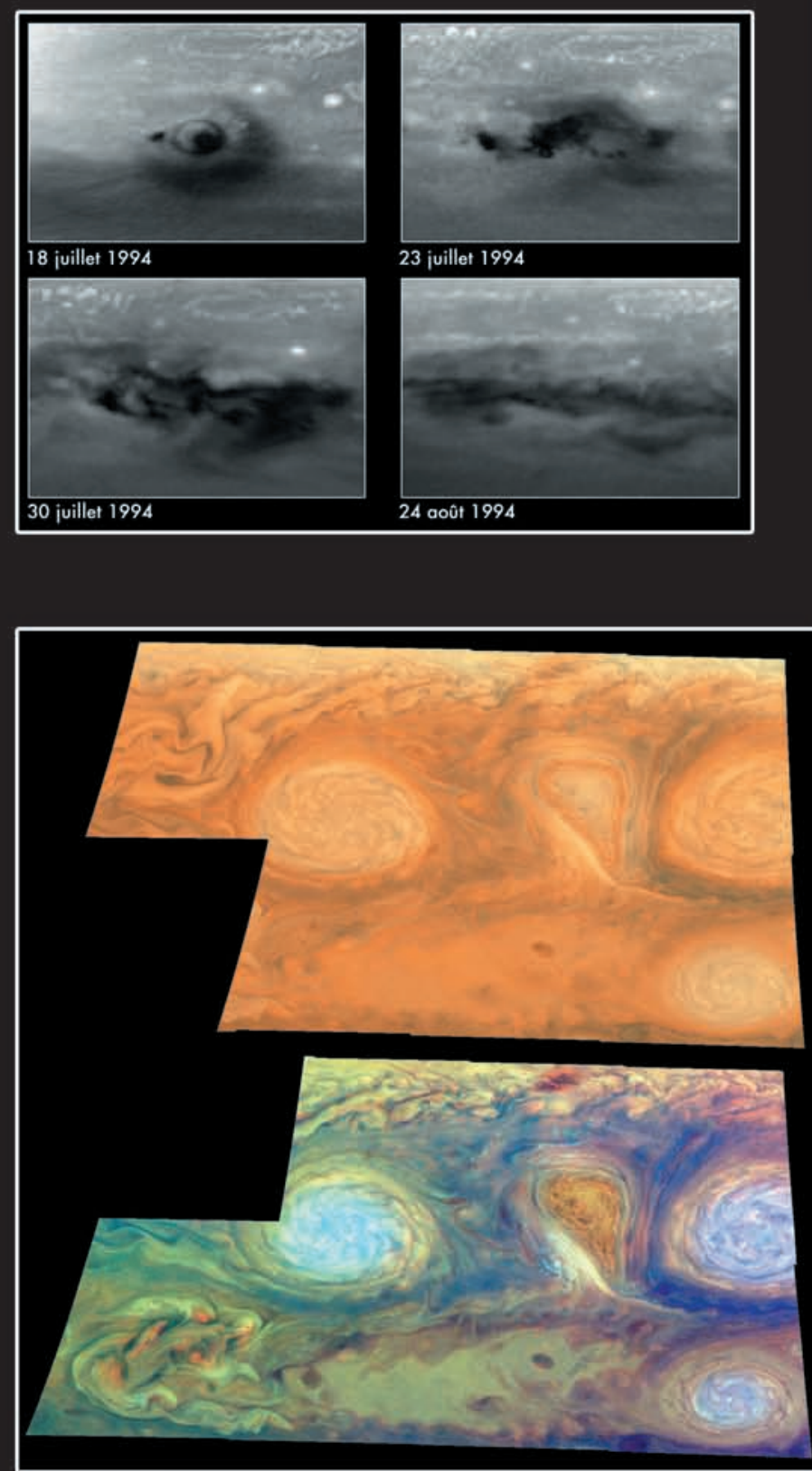

Image des bandes de Jupiter dans l'hémisphère Nord (entre 10 et 50 degrés' de latitude). À gauche, couleurs vraies. À droite, fausses couleurs qui révèlent en bleu les nuages fins, en rouge les nuages profonds, en blanc les nuages épais et élevés, en violet une brume de haute altitude recouvrant une atmosphère claire. (๑ Galileo/Nasa)

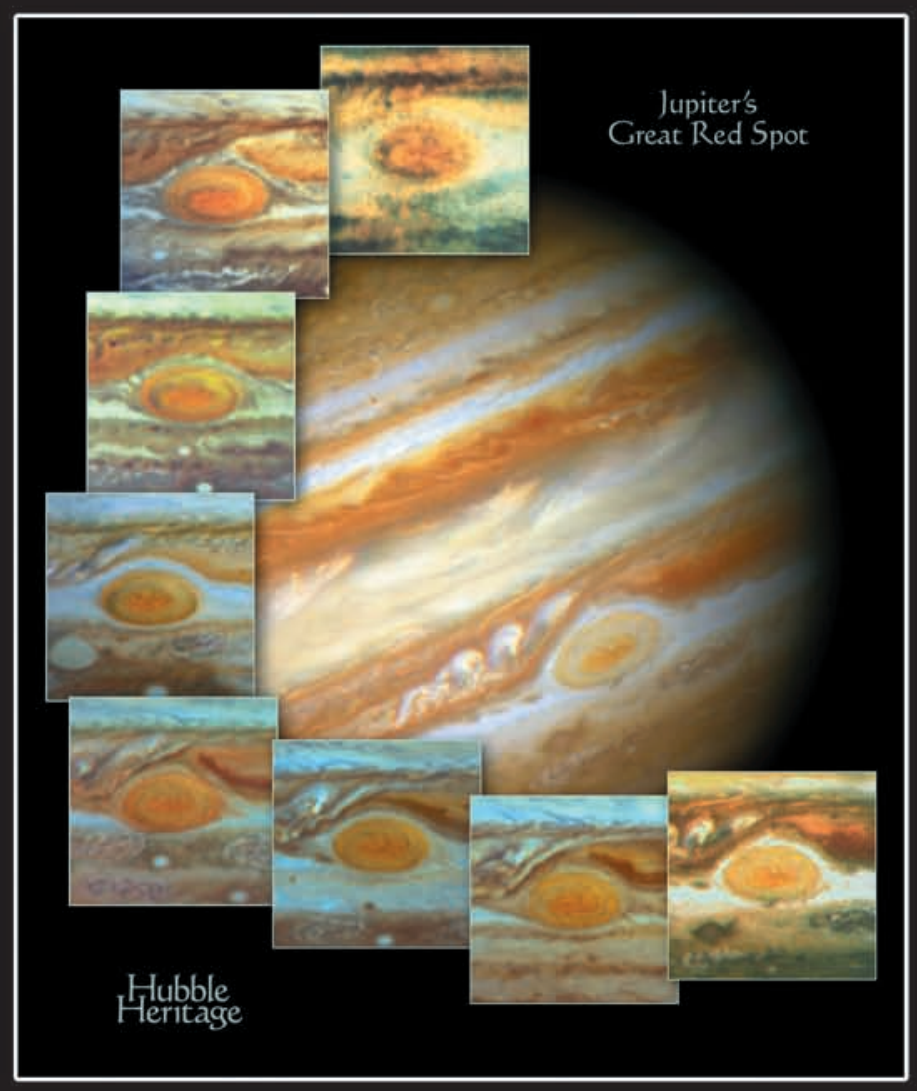

Différents clichés de la tache rouge obtenus par le télescope spatial Hubble entre 1994 et 2004. La forme et la couleur de la tache rouge évoluent au cours du temps. (@) Hubble Heritage Team, STScl/Aura/Nasa/ESA, et Amy Simon, Cornell U.)

En 1994, les différents fragments de la comète Shoemaker-Levy 9 se sont abîmés dans l'atmosphère de Jupiter pour y exploser. Le télescope spatial Hubble dévoile les traces de l'une des explosions et leur dispersion dans l'atmosphère de Jupiter. (๑ H. Hammel, MIT et Nasa/ESA)

Image de deux ovales blancs anticycloniques de l'hémisphère sud et de la dépression coincée entre les deux. En haut, couleurs vraies. En bas, fausses couleurs qui révèlent en bleu les nuages fins,

en rouge les nuages profonds et en blanc les nuages épais et élevés. (C) Galileo/Nasa) 
détruit le méthane vers des altitudes de $300 \mathrm{~km}$ (pression de $10^{-3} \mathrm{hPa}$ ), pour former des molécules plus complexes comme l'éthane $\left(\mathrm{C}_{2} \mathrm{H}_{6}\right)$ et l'acétylène $\left(\mathrm{C}_{2} \mathrm{H}_{2}\right)$ pour les deux plus abondantes, ou le benzène $\left(\mathrm{C}_{6} \mathrm{H}_{6}\right)$ pour la plus complexe. Ces hydrocarbures absorbent très bien le rayonnement ultraviolet, mais sont également de bons « radiateurs » infrarouges. Ils interviennent donc dans le bilan thermique de la haute atmosphère de Jupiter.

\section{Le rôle des comètes}

Enfin, en 1994, la collision de la comète Shoemaker-Levy 9 avec Jupiter a induit une " chimie de choc » qui a synthétisé des molécules inédites, telles que CS ou $\mathrm{HCN}$; un panache, identique à ceux des explosions atomiques, les a déposées vers quelques centaines de kilomètres d'altitude. Ces molécules, bien que lentement diffusées ou détruites, sont toujours observées à l'heure actuelle. Du fait de sa masse imposante, Jupiter attire efficacement les comètes et les collisions y seraient nombreuses, de l'ordre d'une par siècle. Sur le long terme, ce taux de collisions pourrait constituer un flux permanent et continu de matière extrajovienne qui modifierait subtilement l'équilibre chimique de la haute atmosphère de Jupiter.

\section{Les couches nuageuses et le problème de l'eau}

Trois gaz sont susceptibles de condenser et de donner naissance aux couches nuageuses observées : $\mathrm{H}_{2} \mathrm{O}$ à une altitude d'environ $5000 \mathrm{hPa}, \mathrm{H}_{2} \mathrm{~S}$ qui réagit avec $\mathrm{NH}_{3}$ pour former $\mathrm{NH}_{4} \mathrm{SH}$ vers $2000 \mathrm{hPa}$, et enfin $\mathrm{NH}_{3}$ vers $1000 \mathrm{hPa}$. $\mathrm{C}$ 'est la structure canonique des nuages joviens. Dans le détail, beaucoup de questions restent ouvertes. En premier lieu, les nuages sont opaques et empêchent de sonder les masses d'air situées sous leur base. Par télédétection, on ne peut mesurer la pression partielle des gaz condensables qu'au-dessus des nuages, à des altitudes où celle-ci est toujours inférieure à leur pression de vapeur saturante. Ainsi, par télédétection, il est délicat de connaître l'abondance globale de $\mathrm{NH}_{3}$, voire impossible pour celles de $\mathrm{H}_{2} \mathrm{~S}$ et $\mathrm{H}_{2} \mathrm{O}$. Sans ces valeurs, les modèles de nuages demeuraient plus qualitatifs que quantitatifs.

Il a fallu attendre 1995 et le spectromètre de masse de la sonde Galileo pour connaître les fractions molaires de $\mathrm{NH}_{3}$ et $\mathrm{H}_{2} \mathrm{~S}$ (tableau 1). La sonde a également obtenu une mesure pour $\mathrm{H}_{2} \mathrm{O}$, mais il y a tout lieu de croire que celle-ci ne représente pas la valeur globale de la planète. En effet, la sonde est descendue dans une région très particulière, extrêmement sèche, presque dépourvue de nuages, où la pression partielle des gaz condensables est très sous-saturée. La fraction molaire des trois gaz condensables a crû au cours de la descente jusqu'à des pressions beaucoup plus élevées que les pressions de condensation attendues : $8000 \mathrm{hPa}$ pour $\mathrm{NH}_{3}$ au lieu de $1000 \mathrm{hPa}, 14000$ $\mathrm{hPa}$ pour $\mathrm{H}_{2} \mathrm{~S}$ au lieu de $2000 \mathrm{hPa}$. L'abondance de l'eau était toujours en train de croître lorsque la sonde a cessé de fonctionner, vers $22000 \mathrm{hPa}$. La dernière mesure ne représente donc pas la valeur profonde, globale de $\mathrm{H}_{2} \mathrm{O}$. Quelle est alors cette valeur?

Plusieurs réponses indirectes sont proposées :

- La première est théorique. Elle consiste à comparer les abondances relatives de $\mathrm{C} / \mathrm{H}, \mathrm{N} / \mathrm{H}, \mathrm{S} / \mathrm{H}, \mathrm{Ar} / \mathrm{H}, \mathrm{Xe} / \mathrm{H}$ et $\mathrm{Kr} / \mathrm{H}$ dans Jupiter à leurs abondances relatives dans le Soleil. On s'aperçoit alors que les abondances relatives joviennes sont en moyenne trois fois plus élevées que les abondances relatives solaires. Cet enrichissement est expliqué par le modèle le plus probable de formation de Jupiter dans la nébuleuse protosolaire (Lunine et al., 2004) : un noyau composé de glaces riches en éléments lourds $(\mathrm{O}, \mathrm{C}, \mathrm{N}, \mathrm{S} \ldots)$ s'est constitué pour atteindre une masse critique d'environ 15 à 20 masses terrestres. Cette masse atteinte, le noyau a capturé par gravitation le gaz léger $\left(\mathrm{H}_{2}\right.$ et $\mathrm{He}$ ) de la nébuleuse pour constituer l'atmosphère de la planète. Le noyau glacé s'est partiellement ou totalement dissous dans l'enveloppe gazeuse pour l'enrichir en C, N, S... L'oxygène, et par là l'eau, doit donc être également enrichi, les modèles de formation prévoyant un rapport $\mathrm{O} / \mathrm{H}$ jovien de trois à dix fois le rapport solaire.

- Comme mentionné ci-dessus, on observe une molécule d'origine interne, $\mathrm{CO}$, qui n'est que partiellement transformée en $\mathrm{CH}_{4}$ dans l'atmosphère superficielle. À partir d'hypothèses sur le temps de mélange dynamique de l'atmosphère et sur la cinétique de la réaction, il est possible d'obtenir une valeur du rapport $\mathrm{O} / \mathrm{H}$ dans Jupiter : cette valeur représente trois fois le rapport solaire, mais l'incertitude est telle que cette valeur peut varier entre 1 et 10 fois le rapport solaire (Bézard et al., 2002).

- Les sondes spatiales qui sont passées à proximité de Jupiter ont mesuré son champ de gravitation. Celui-ci n'est pas à symétrie sphérique, du fait de l'aplatissement de la planète. La mesure de l'écart à la sphéricité permet de déduire la distribution des masses à l'intérieur de la planète et, en particulier, la masse totale des éléments plus lourds que l'hydrogène et l'hélium. Une telle analyse montre que les éléments lourds sont enrichis entre 1,5 et 6 fois leur abondance solaire (Guillot et al., 2004).

Ainsi, plusieurs méthodes indépendantes tendent à montrer que le rapport $\mathrm{O} / \mathrm{H}$ doit être élevé dans Jupiter, à savoir probablement au moins trois fois le rapport solaire. Mais on ne connaît pas la valeur exacte. Cela induit des incertitudes sur la pression de la base de la couche nuageuse d'eau, mais également sur la dynamique. Dans une cellule de convection, lorsque l'air s'élève, la température décroît par détente adiabatique. La décroissance de la température provoque la condensation de l'eau et la formation de nuages (sur Terre, les cumulus ou les cumulonimbus). La condensation de l'eau relâche de la chaleur latente qui réchauffe la masse d'air et amplifie le phénomène de convection (l'air chaud monte plus efficacement). Sur Jupiter, l'eau est la plus abondante des molécules condensables et celle qui possède la chaleur latente la plus élevée. Ne pas connaître son abondance constitue donc un problème majeur pour notre compréhension de la météorologie et de la dynamique joviennes.

\section{L'ammoniac et la couleur des nuages}

D'après le modèle théorique de la structure verticale des nuages joviens, la couche nuageuse extérieure, la plus élevée en altitude, doit être constituée d'ammoniac. Les contrastes à la surface de la planète doivent donc résulter des variations spatiales de l'altitude et de l'épaisseur de cette couche nuageuse. Toutefois, un problème se pose : la glace d'ammoniac est facilement détectable par spectroscopie mais, jusqu'en 2002, elle est restée cachée aux observateurs. Grâce aux spectres et aux images à haute résolution spatiale fournis par la sonde Galileo, Baines et al. (2002) ont pu montrer que la glace d'ammoniac existe, mais seulement en quelques régions très localisées où elle est associée à des nuages de haute altitude, certainement le sommet de cellules convectives (figure 4). Quelle est alors la nature chimique des nuages joviens sur le reste de la planète ? D'où proviennent leurs différences de couleur? Ces questions sont ouvertes, et on ne peut avancer que des hypothèses. La plus plausible est que la glace d'ammoniac évolue, sous l'effet du rayonnement 
Figure 4 - Image en fausses couleurs de la grande tache rouge montrant sur son flanc nord-ouest un nuage riche en glace d'ammoniac (zone bleutée). (D'après Baines et al., 2002, (c) Nasa/Galileo)

ultraviolet solaire, vers d'autres formes, comme l'hydrazine $\left(\mathrm{N}_{2} \mathrm{H}_{4}\right)$. La photodissociation d'autres molécules, en particulier la phosphine, peut également produire des molécules colorées appelées chromophores, par exemple le phosphore rouge $\left(\mathrm{P}_{4}\right)$. Mais, jusqu'à présent, ces composés hypothétiques restent indécelables.

\section{La structure thermique de l'atmosphère}

La structure thermique de l'atmosphère jovienne peut être déterminée par trois méthodes :

- Les émissions de $\mathrm{H}_{2}$ et de $\mathrm{CH}_{4}$ dans l'infrarouge thermique permettent de sonder la température entre 100 et $500 \mathrm{hPa}$, d'une part, et entre 1 et $10 \mathrm{hPa}$, de l'autre.

- Les occultations stellaires sondent entre $10^{-3} \mathrm{hPa}$ et $10^{-1} \mathrm{hPa}$, tandis que les occultations du signal radio émis par les missions spatiales sondent entre $10^{-2} \mathrm{hPa}$ et $1000 \mathrm{hPa}$

- La sonde de descente Galileo a mesuré le profil de température in situ entre $10^{-5} \mathrm{hPa}$ et $22000 \mathrm{hPa}$.

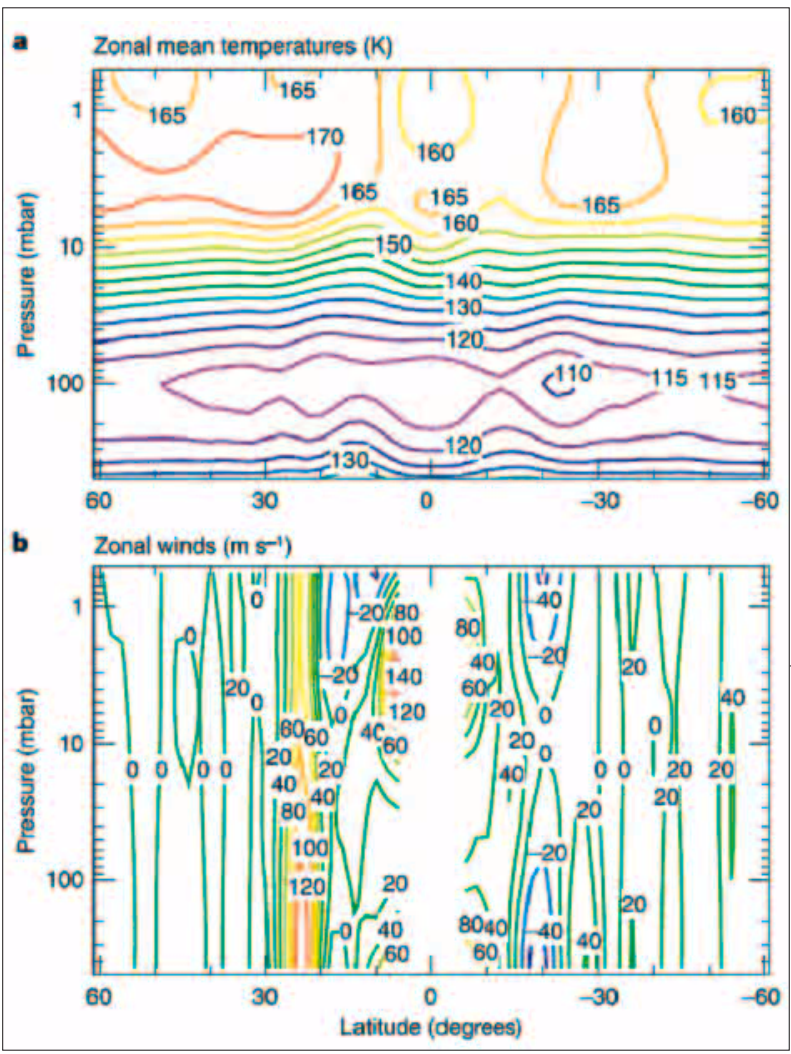

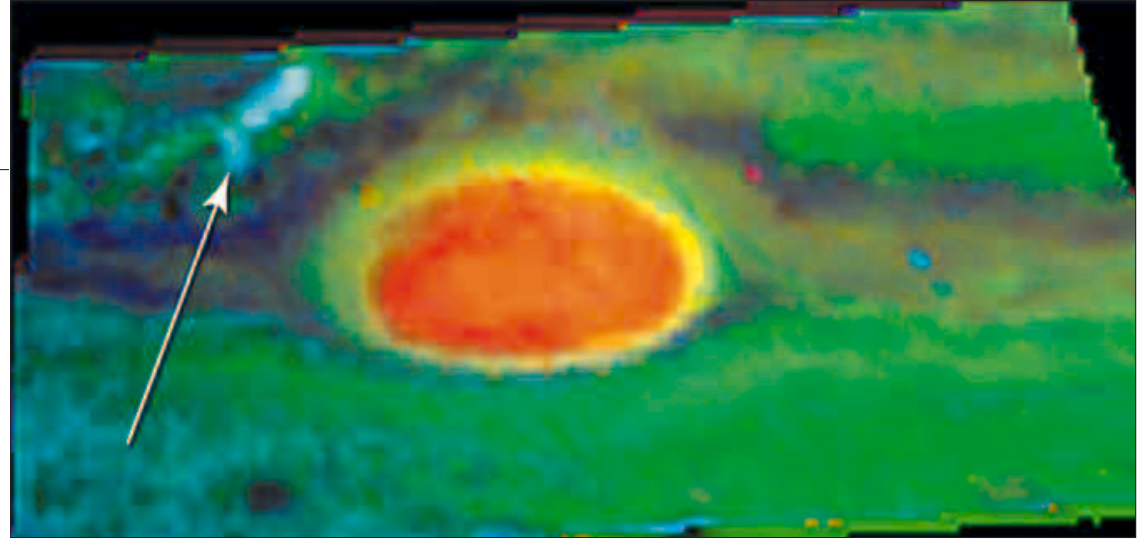

La télédétection permet d'obtenir des cartes de la température, mais sa capacité de résolution verticale est limitée (typiquement à une demi-échelle de hauteur). Les occultations et le sondage in situ fournissent une haute résolution verticale, mais en un nombre limité de points géographiques. Enfin, seules les mesures in situ sondent les pressions supérieures à $1000 \mathrm{hPa}$.

Le profil vertical moyen de la température (figure 5) fait apparaître une troposphère (région où la température décroît avec l'altitude), possédant une tropopause de $110 \mathrm{~K}$ en moyenne, localisée vers $100 \mathrm{hPa}$. Aux pressions inférieures, existe une stratosphère (région où la température augmente avec l'altitude) : la température y atteint $160-170 \mathrm{~K}$ vers 2-3 $\mathrm{hPa}$. Le réchauffement y est induit par l'absorption du rayonnement ultraviolet solaire par le méthane et les hydrocarbures plus lourds. Entre $1 \mathrm{hPa}$ et $10^{-3} \mathrm{hPa}$, la température reste relativement constante. Dans cette gamme d'altitudes, l'absorption du flux solaire est compensée par le rayonnement infrarouge des hydrocarbures. Aux pressions inférieures à $10^{-3} \mathrm{hPa}$, la diffusion turbulente devient négligeable

Figure 5 - Coupe verticale méridienne de la température (en haut) et du vent zonal (en bas) en fonction de la latitude et de la pression, tels qu'observés par le spectromètre Cassini/CIRS (D'après Flasar et al., 2004 avec l'autorisation de MacMillan Publishers Ltd : Nature, (C) 2004) devant la diffusion moléculaire. Au-delà de cette limite appelée homopause, les molécules se séparent en fonction de leur masse : les hydrocarbures lourds, radiateurs infrarouges, disparaissant, l'atmosphère se réchauffe. Toutefois, l'amplitude du réchauffement ne peut s'expliquer seulement par l'absorption du flux solaire par $\mathrm{H}_{2}$; une autre source de chaleur doit exister. Deux possibilités sont avancées : la dissipation d'ondes thermiques et les précipitations de particules chargées accélérées dans le champ magnétique de la planète ; mais le bilan énergétique reste à clarifier.

\section{La troposphère}

Les cartes de température dans la troposphère frappent tout d'abord par l'absence d'un gradient entre l'équateur et les pôles. Soit il existe un mécanisme de redistribution vers les pôles de l'énergie solaire absorbée préférentiellement à l'équateur, soit la source d'énergie interne due au lent refroidissement du cœur de la planète compense les gradients d'énergie solaire. Les anomalies troposphériques de température sont corrélées à la structure en zones et en ceintures : les zones sont plus froides que les ceintures. Ces anomalies de température permettent de contraindre l'évolution des vents avec l'altitude (via l'équation du vent thermique). En effet, les ceintures dépressionnaires chaudes voient leur pression décroître plus lentement avec l'altitude que les zones anticycloniques froides. Dans la haute troposphère, le contraste de pression entre zones et ceintures se réduit, la vitesse des vents s'atténue (figure 5). Ce phénomène est connu sur Terre, où les structures chaudes et dépressionnaires au sol deviennent légèrement anticycloniques en altitude. Ainsi, sur Jupiter, les anomalies de température observées impliquent que les vents doivent décrô̂tre avec l'altitude au-dessus de la couverture nuageuse. 
Figure 6 - Image des aurores de Jupiter obtenue dans l'ultraviolet par le télescope spatial Hubble. La structure complexe, correspond à différentes sources. L'aurore comprend un ovale principal, centré sur le pôle nord pour cette image. Les émissions diffuses à l'intérieur de l'ovale correspondent à une entrée du vent solaire. À l'extérieur de l'ovale, trois points lumineux sont visibles : l'un à l'extrême gauche, deux côte à côte légèrement sur la droite de l'image. Ils correspondent aux pieds des tubes de courant électrique qui relient, le long des lignes de champ magnétique, les satellites lo, Europe et Ganymède à l'atmosphère de Jupiter. (C) Nasa)

\section{La stratosphère}

Dans la stratosphère, la structure « zones-ceintures » disparaît, ou du moins les anomalies positives et négatives de température par rapport à la température moyenne ne sont pas corrélées aux structures troposphériques. La haute stratosphère est particulièrement chaude aux hautes latitudes, avec des températures qui peuvent atteindre $200 \mathrm{~K}$. Ce réchauffement est induit par les précipitations aurorales joviennes, extrêmement énergétiques. Leur puissance moyenne, $10^{14} \mathrm{~W}$, est comparable à la puissance du rayonnement ultraviolet solaire absorbée par Jupiter. Mais, contrairement au rayonnement solaire, les précipitations aurorales sont concentrées sur d'étroites régions proches des pôles (figure 6). Ces précipitations aurorales doivent aussi induire une chimie particulière, encore mal comprise.

De nombreuses ondes de densité et de température ont été révélées par les occultations et le sondage in situ de la stratosphère jovienne. L'amplitude des ondes varie de 5 à $25 \mathrm{~K}$, tandis que leur longueur d'onde verticale est de l'ordre d'une échelle de hauteur et demie. Sur les cartes de température stratosphérique, la mission Cassini a mis en évidence la propagation d'une anomalie de température négative située vers $4 \mathrm{hPa}$, vraisemblablement associée à une onde. La nature des ondes observées est encore débattue. En tout état de cause, nous pensons que celles-ci influent sur la météorologie jovienne. Tous les quatre ans environ, autour de l'équateur, la haute troposphère (300-100 hPa) et la moyenne stratosphère $(3 \mathrm{hPa})$ se refroidissent par rapport à leur température

Figure 7 - Image, enregistrée par Galileo de la grande tache rouge de Jupite et des nuages convectifs situés de part et d'autre. Les couleurs donnent l'altitude des nuages

en blanc, nuages élevés ; en bleu, nuages profonds.

Les deux images du bas sont un zoom de la région encadrée sur l'image du haut

elles montrent l'évolution des nuages convectifs entre deux poses espacées de 70 minutes.

(C) Nasa/Galileo)

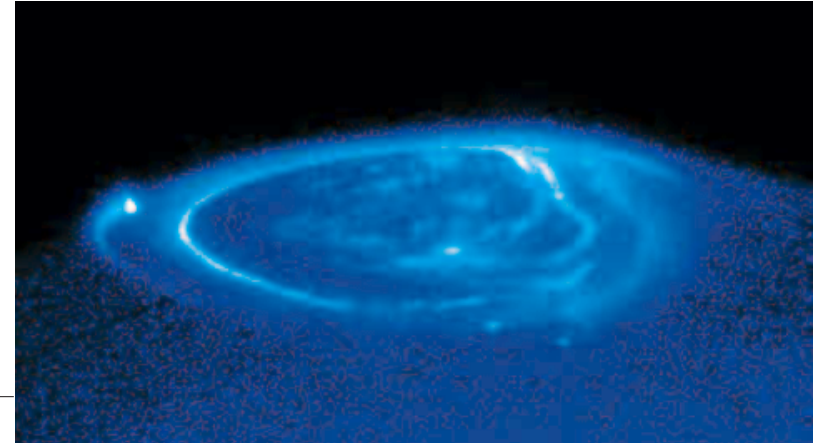

rects. Globalement, les zones apparaissent comme des régions d'ascendance et les ceintures comme de régions de subsidence. Ce schéma est conforté par quatre indices :

- La couverture nuageuse plus épaisse et plus haute, dans les zones, est le signe de

moyenne. Ce phénomène d'oscillation quasi quadriennale semble être la conséquence de l'interaction des ondes avec les vents joviens. Un phénomène analogue, l'oscillation quasi biennale, est bien connu dans l'atmosphère terrestre.

\section{Les mouvements verticaux}

Les capacités actuelles d'observation ne permettent pas, en général, d'observer directement des mouvements verticaux. Pour déterminer leur présence et leur intensité, on a recours à des moyens indi- mouvements ascendants qui entraînent le refroidissement, la saturation et la condensation des espèces condensables.

- Les cartes de l'humidité relative en ammoniac montrent que celle-ci est plus élevée dans les zones que dans les ceintures. L'air monte, humide, dans les zones pour redescendre, sec, dans les ceintures (Gierasch et al., 1986).

- Les zones sont plus froides que les ceintures. Cette différence de température entre les masses d'air montantes et descendantes est bien connue. On peut notamment l'observer sur Terre grâce au phénomène de foehn, ce vent chaud qui se produit dans les plaines, au pied des montagnes, lorsque l'air redescend après avoir franchi les sommets voisins.

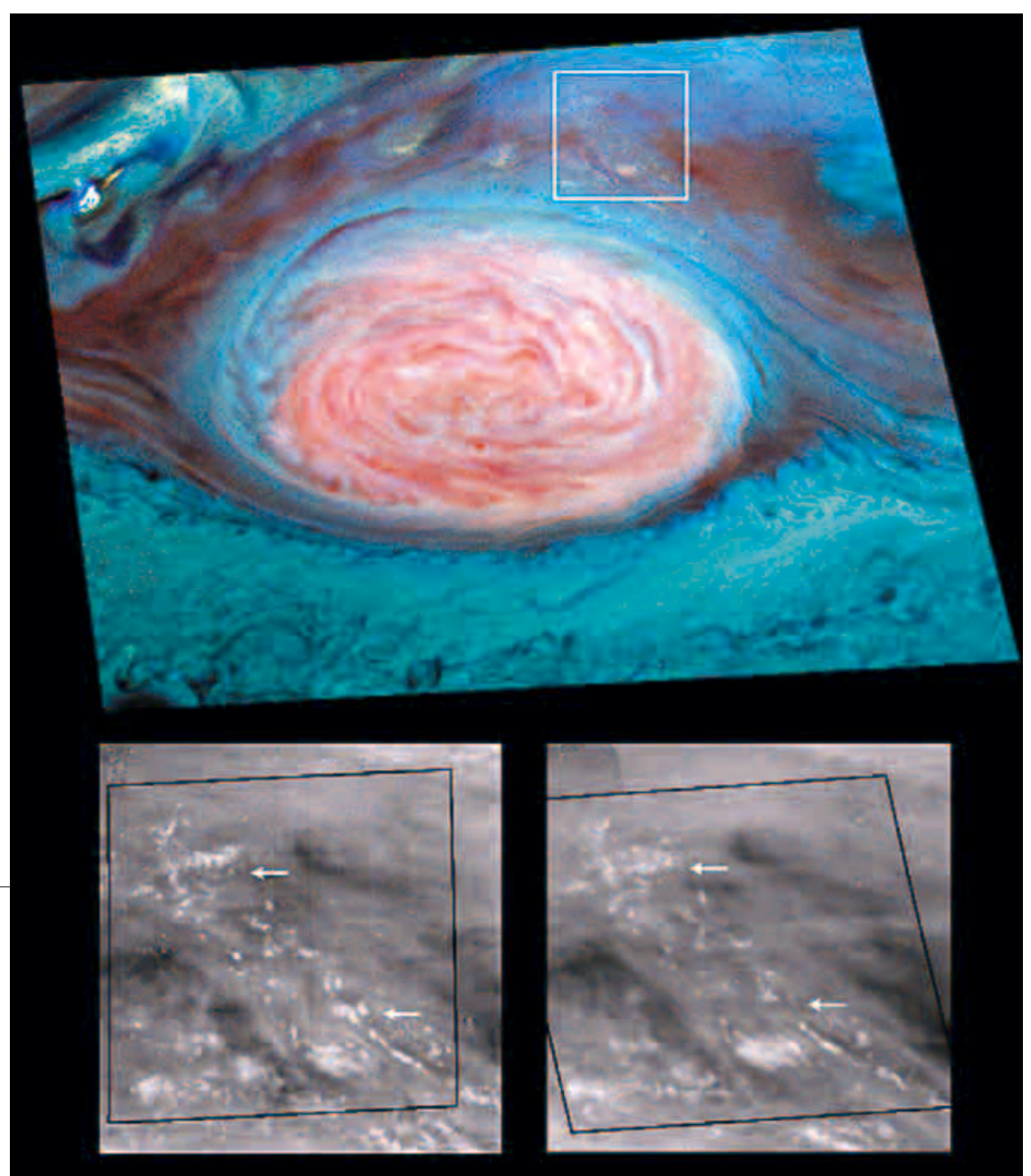


- Le quatrième indice est propre à Jupiter (et aux autres planètes géantes). L'hydrogène moléculaire présente deux états, ortho et para, dont le rapport à l'équilibre thermodynamique varie en fonction de la température et peut être mesuré par télédétection. À $0 \mathrm{~K}$, tout l'hydrogène doit être dans l'état para, puis la fraction de para diminue asymptotiquement jusqu'à 0,25 lorsque la température augmente. Toutefois, la conversion entre les deux états est lente et l'écart à l'équilibre thermodynamique est très probable. Ainsi, si les ceintures apparaissent proches de l'équilibre, les zones sont hors équilibre. La fraction d'hydrogène para $\mathrm{y}$ correspond à celle d'un air plus chaud. $\mathrm{Ce}$ déséquilibre chimique est la signature d'un mouvement ascendant dans les zones, qui est plus rapide que la conversion ortho-para (Conrath et al., 1998).

À partir de ces quatre observations, il est possible d'estimer une vitesse verticale d'ensemble. Elle est extrêmement faible, de l'ordre de $10^{-5} \mathrm{~m} / \mathrm{s}$ dans la région équatoriale. Sur Terre, la vitesse verticale moyenne dans les régions tropicales d'ascendance est de quelques millimètres par seconde. Cette comparaison montre clairement que les échelles de temps des mouvements et des transports verticaux sont extrêmement différentes entre les deux planètes. Jupiter est une planète qui semble immuable comparée à la Terre.

Toutefois, on observe des phénomènes plus violents, mais locaux, de convection humide : il existe, à des endroits bien localisés, des nuages brillants dont le sommet est situé plus haut que le niveau moyen nuageux sur le reste de la planète (figure 7). Ces nuages possèdent plusieurs caractéristiques qui les rapprochent des cumulonimbus terrestres. La spectroscopie fait apparaître que ce sont les régions les plus riches en eau que l'on puisse observer, avec une humidité relative de $40 \%$. Ce sont également les seuls nuages où la glace d'ammoniac soit visible (figure 4), ce qui prouve que la convection y est plus rapide que l'altération photochimique. Enfin, lorsque la caméra à bord de Galileo observe de tels nuages disparaître dans la nuit jovienne, les images de nuit révèlent de multiples éclairs (figure 8). Ces éclairs se produisent profondément à l'intérieur du nuage et les photons sont diffusés jusqu'à son sommet. La largeur de la tache due à un éclair, d'environ 60 à 120 $\mathrm{km}$, est équivalente à sa profondeur. Cela correspond à un niveau de pression de $8000 \mathrm{hPa}$, où seule l'eau peut condenser.
Figure 8 - Éclairs dans la nuit jovienne. Les nuages sont

faiblement éclairés par un clair de lo. Image obtenue par la sonde Galileo. (C) Nasa)

On observe ainsi des colonnes nuageuses très épaisses, de 8000 à quelques centaines d'hectopascals, humides et d'ascension rapide, associées à de la convection humide. La figure 2 montre l'emplacement de ces structures, présentes uniquement dans les ceintures, normale-

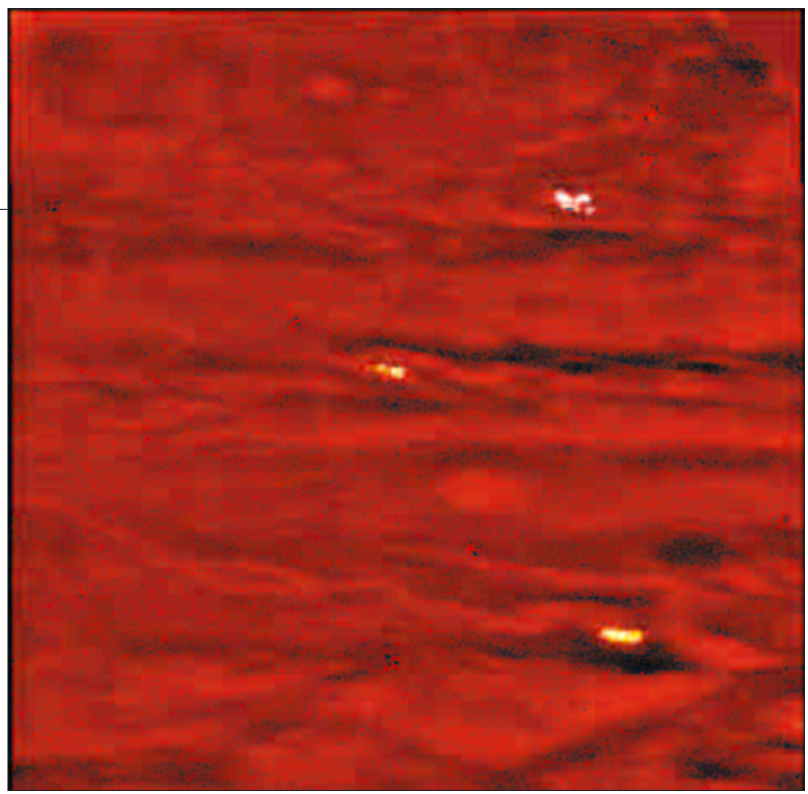
ment associées à des mouvements descendants. Ce phénomène est d'autant plus important que certains auteurs pensent que la moitié de l'énergie libérée au cœur de la planète, par de lents refroidissements et par contraction, s'échappe dans l'atmosphère via ces nuages convectifs. Mais intégrer cette observation surprenante dans notre schéma actuel de la circulation générale de Jupiter n'est pas chose aisée.

\section{Modèles dynamiques et météorologiques de Jupiter}

Les modèles dynamiques de Jupiter tentent d'expliquer, au moins qualitativement, les observations météorologiques que nous venons de décrire. Ils doivent répondre à trois grandes questions :

- Quelle est l'origine de la structure en bandes et quelles sont les raisons de sa stabilité ?

Figure 9 - Schéma du modèle de circulation profonde de Jupiter. La circulation s'organise en colonnes en rotation. Ces colonnes s'assemblent en cylindres concentriques et parallèles, de sens de rotation alternés. À l'intersection avec

la couverture nuageuse, les cylindres donnent naissance à des courants-jets soufflant en alternance d'est en ouest et d'ouest en est. (C) Augustin Sanchez Lavega)
- Pourquoi l'équateur de Jupiter tournet-il plus vite que le reste de l'atmosphère et que le champ magnétique de la planète ? Comment cet excès de moment cinétique se maintient-il au cours du temps?

- Quelle est l'origine des tourbillons et pourquoi sont-ils si stables?

Les modèles se regroupent en deux grandes catégories : les modèles profonds et les modèles en couche limite.

\section{Les modèles profonds}

Les modèles profonds montrent qu'une sphère de gaz en rotation rapide, chauffée de l'intérieur, tend à s'organiser sous forme de colonnes en rotation. Ces colonnes s'assemblent en cylindres concentriques et parallèles, convectifs, de sens de rotation alterné (figure 9). À

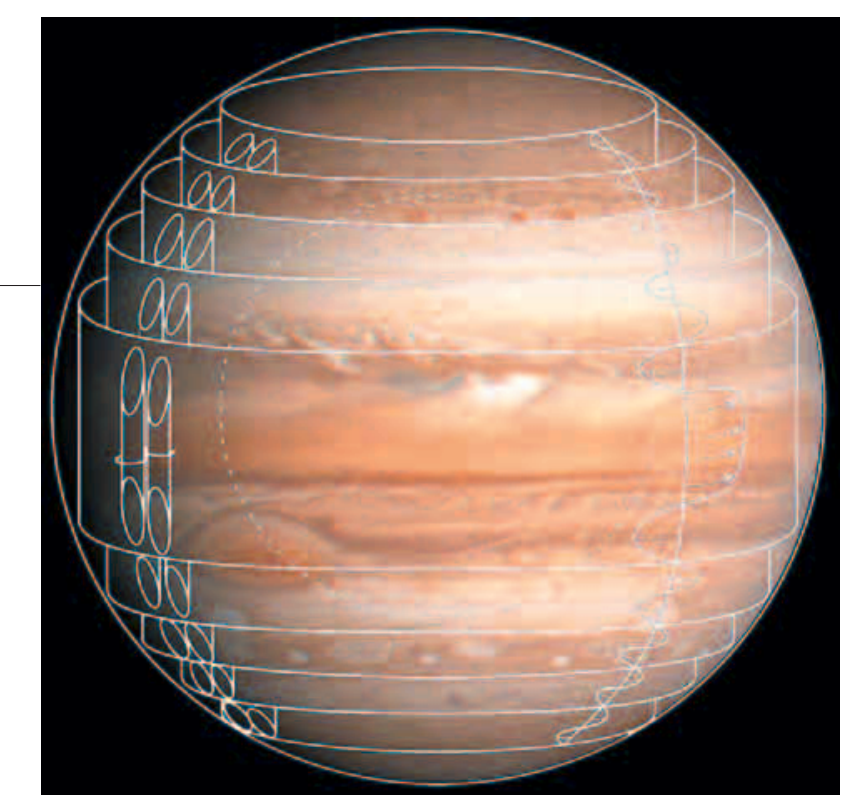


l'intersection avec les couches observables depuis l'espace, ces cylindres donnent naissance à des courants-jets soufflant en alternance d'est en ouest et d'ouest en est. Ce type de modèle reproduit donc avec succès la structure jovienne en bandes, telle que nous l'observons, et explique sa haute symétrie. L'inertie des cylindres qui traversent la planète de part en part est suffisamment grande pour assurer leur stabilité sur le long terme. Un autre succès pour cette théorie a été la mesure des vents joviens en profondeur (22 $000 \mathrm{hPa}$ ) par la sonde Galileo, qui a montré que ceux-ci croissent jusque vers $5000 \mathrm{hPa}$, puis restent uniformes.

Toutefois, ce type de modèle se heurte au problème de l'état de l'intérieur de la planète. Aux hautes pressions de l'ordre de $10^{9} \mathrm{hPa}$, l'hydrogène doit subir une transition de phase entre hydrogène moléculaire et hydrogène métallique. Comment des cylindres en rotation différentielle pourraient-ils pénétrer dans ce cœur métallique, en rotation rigide sous l'influence du champ magnétique ? Il existerait donc une latitude limite, au-delà de laquelle la structure zonale de Jupiter devrait disparaître. Or, on observe une structure globale à tendance zonale jusque vers 80 degrés de latitude nord et sud.

Quantitativement, les simulations deviennent plus difficiles à confronter aux observations. Les différents auteurs annoncent que les modèles profonds donnent des vents à l'équateur compris entre de très faibles valeurs et $70 \mathrm{~m} / \mathrm{s}$. La latitude des frontières entre zones et ceintures, le nombre de ceintures et de zones dans chaque hémisphère restent inexplicables avec les simulations numériques actuelles. Les modèles ne simulent pas non plus l'intensité des mouvements convectifs verticaux.

\section{Les modèles en couche limite}

Cette classe de modèles fait naître les structures dans l'atmosphère superficielle de la planète (vers quelques millions de pascals) sous l'effet de

Séquence de neuf images (en vraies couleurs montrant comment varie l'apparence de Jupiter lorsqu'il effectue une rotation complète sur lui-même. Jupiter effectue une rotation en à peu près 10 heures. Images prises les 22 et 23 octobre 2000 par la sonde Cassini. (C) Nasa/JPL/University of Arizona) l'inégale répartition de l'irradiation solaire. Pour une planète en rotation rapide (approximation quasi géostrophique), les structures tourbillonnaires à petite échelle se regroupent au cours d'une cascade vers les grandes structures, pour finalement établir le régime de vents zonaux que l'on observe. Un aspect important est que cette cascade de tourbillons tend à concentrer le moment cinétique à l'équateur et, donc, à expliquer la rotation rapide de la zone équatoriale de Jupiter. Ce type de modèle est également capable d'expliquer la forme des tourbillons joviens, elliptiques, et leur tendance à fusionner.

Mais les difficultés ne manquent pas non plus pour cette classe de modèles. En effet, ils simulent des zones et des ceintures beaucoup plus larges que la réalité, et dans lesquelles les vents doivent être plus faibles que ceux mesurés. De plus, il doit exister un gradient de température d'une dizaine de degrés de l'équateur vers les pôles pour que ce régime de circulation puisse s'établir. Or, un tel gradient est absent, du moins dans les couches observables. Enfin, les modèles en couche limite, pas plus que les modèles profonds d'ailleurs, ne parviennent pour l'instant à simuler l'évolution des tourbillons joviens. Pourquoi et quand des tourbillons qui ont cohabité pendant des dizaines d'années fusionnent-ils ? Pourquoi certains deviennent instables et disparaissent? Cela pourrait être en particulier le cas de la grande tache rouge : depuis les années 1900, elle tend à devenir circulaire, donc instable. Le plus vieux des tourbillons joviens pourrait donc disparaître sous nos yeux, sans que nous comprenions réellement le moteur de sa disparition.

\section{Conclusions}

Nous devons donc, pour l'instant, avouer notre impuissance à établir un modèle cohérent et global de la météorologie et de la dynamique joviennes. D'autant que des observations récentes viennent de mettre à mal quelques faits que l'on pensait acquis. Ainsi, sur Saturne, on vient de s'apercevoir que l'on n'avait pas accès de manière fiable à la période de rotation de la planète (voir encadré page 29). Le problème pourrait être similaire sur Jupiter. En outre, la prépondérance des nuages violemment convectifs dans les ceintures a conduit certains auteurs (Showman et De Pater, 2005) à proposer que, sous les couches nuageuses, les ceintures soient des régions de mouvements ascensionnels, à l'exact opposé de ce qui est observé au-dessus des nuages. Ces nouvelles

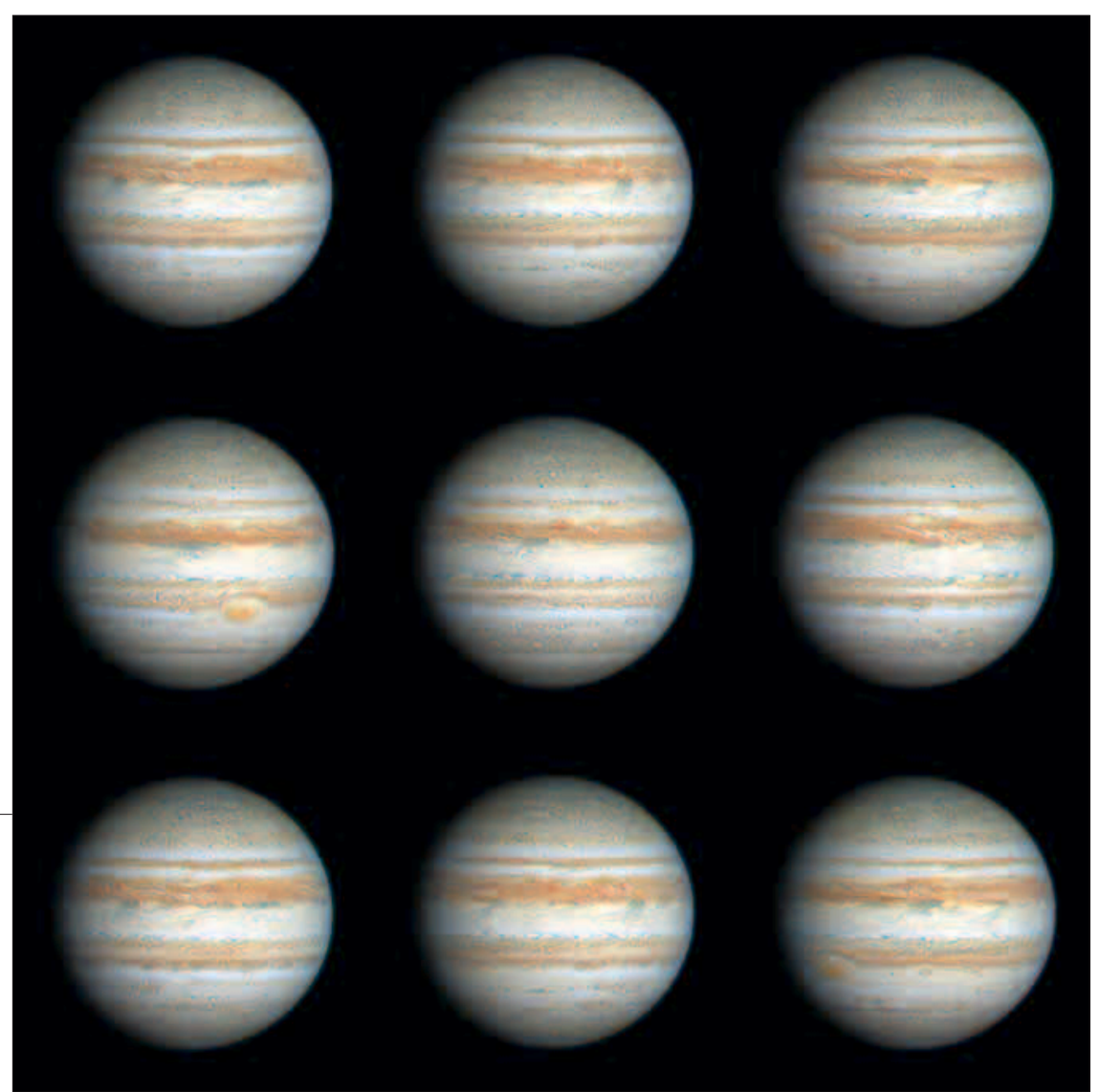


observations et spéculations pointent très précisément les lacunes actuelles : on ne connaît rien ou presque des vents, de la composition chimique et du champ de température à des pressions supérieures à $1000 \mathrm{hPa}$. C'est pourquoi la plupart des projets d'exploration de Jupiter mettent l'accent sur :

\section{Quelle est la durée du jour sur Saturne?}

Mesurer la période de rotation de Saturne (comme celle de toutes les planètes géantes) est un problème délicat. En effet, il n'existe pas de surface rigide accessible, tandis que l'atmosphère présente une rotation différentielle. Dans ces conditions, on prend comme référence la période de rotation du champ magnétique, qui est liée à celle de l'ensemble de la planète via son intérieur métallique et conducteur. La période de rotation du champ magnétique est elle-même mesurée grâce aux émissions radio kilométriques, inaccessibles depuis la Terre. La sonde Voyager les avait

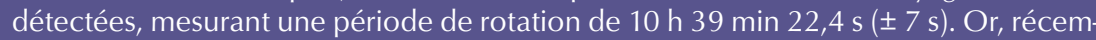
ment, les missions Ulysse et Cassini ont montré que la période des émissions kilométriques de Saturne s'est allongée à 10 h 45 min $45 \mathrm{~s}$ ( $\pm 36 \mathrm{~s}$ ).

Étant donné le moment cinétique de Saturne, il est hors de question que la période de rotation ait changé. Cet événement suggère plutôt que l'on ne comprend pas correctement le processus d'émission des ondes radio et leur lien avec le champ magnétique. Se pose aussi la question de savoir si le champ magnétique est bien ancré avec la rotation du cœur interne de la planète. En attendant la réponse à ces questions, l'incertitude sur la période de rotation de Saturne pose de nombreux problèmes aux planétologues et aux astrophysiciens. Quelle est la vitesse réelle des vents sur Saturne, mais aussi sur les autres planètes géantes, y compris Jupiter?

Image de Saturne obtenue par les caméras de la sonde Cassini en mai 2004 (C Nasa/JPL/Space Science Institute)

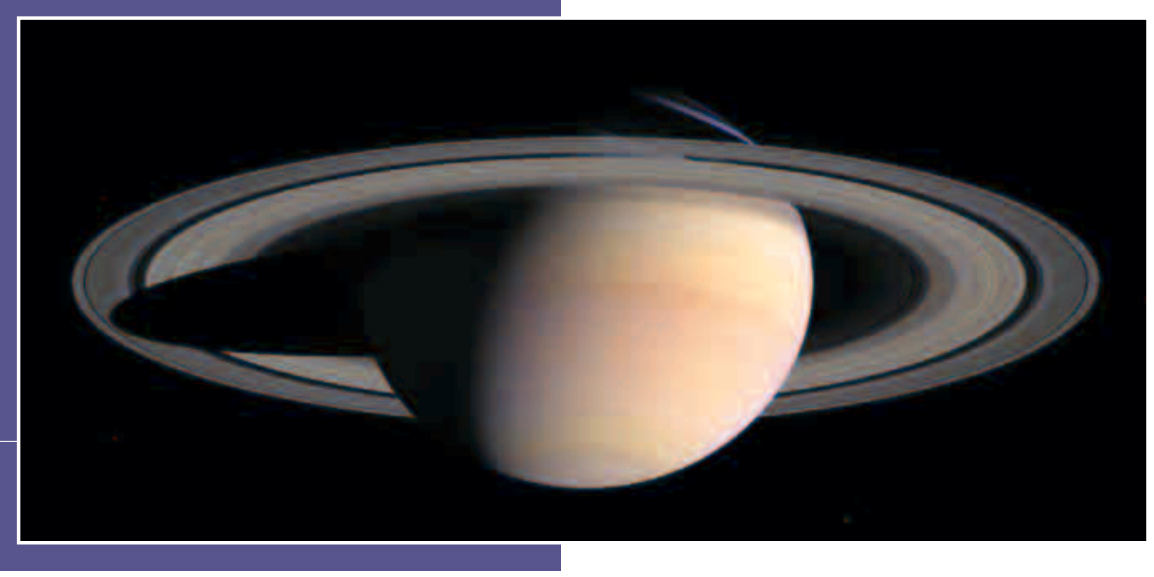

- Le sondage in situ jusqu'à des pressions d'environ $10^{5} \mathrm{hPa}$, et cela en plusieurs points de la planète.

- Le sondage radio qui permettrait de cartographier la quantité d'ammoniac et d'eau ainsi que la température jusque vers $3.10^{4} \mathrm{hPa}$.

- La mesure précise du champ de gravitation ainsi que des mesures en laboratoire de l'équation d'état de l'hydrogène aux hautes pressions, afin de mieux connaître l'état de l'intérieur de la planète.

Pendant ce temps, théoriciens et modélisateurs travailleront à mieux mettre en équations et à mieux modéliser numériquement la complexité d'une atmosphère profonde de plusieurs dizaines de milliers de kilomètres.

\section{Bibliographie}

\footnotetext{
- Baines et al., 2002 : Fresh ammonia ice clouds in Jupiter. I. Spectroscopic identification, spatial distribution, and dynamical implications. Icarus, 159 , 74-94.

- Bézard B., E. Lellouch, D. Strobel, J.-P. Maillard et P. Drossart, 2002 : Carbon monoxide on Jupiter: Evidence for both internal and external sources. Icarus, $159,95-111$.

- Conrath B. J., P. J. Gierasch et E. A. Ustinov, 1998 : Thermal structure and para hydrogen fraction on the outer planets from Voyager IRIS measurements. Icarus, $135,501-517$.

- Flasar F. M. et al., 2004 : An intense stratospheric jet on Jupiter. Nature, 427, 132-135.

- Gierasch P. J., J. A. Magalheaes et B. J. Conrath, 1986 : Zonal mean properties of Jupiter's upper troposphere from Voyager infrared observations. Icarus, 67, 456-483.

- Guillot T., D. J. Stevenson, W. B. Hubbard et D. Saumon, 2004 : The interior of Jupiter. In Jupiter. The planet, Satellites and Magnetosphere. Eds F. Bagenal, T. Dowling - et W. McKinnon. Cambridge University Press, 35-57.

- Limaye S. S., 1986 : Jupiter: New estimates of the mean zonal flow at the cloud level. Icarus, 65, 335-352.

- Lunine J. I., A. Coradini, D. Gautier, T. C. Owen et G. Wuchterl, 2004 : The origin of Jupiter. In Jupiter. The planet, Satellites and Magnetosphere. Eds F. Bagenal, - T. Dowling et W. McKinnon. Cambridge University Press, 19-34.

- Showman A. P. et I. De Pater, 2005 : Dynamical implications of Jupiter's tropospheric ammonia abundance. Icarus, 174, $192-204$.

- Taylor F. W., S. K. Atreya, T. Encrenaz, D. M. Hunten, P. G. J. Irwin et T. C. Owen, 2004 : The composition of the atmosphere of Jupiter. In Jupiter. The planet, Satellites and Magnetosphere. Eds F. Bagenal, T. Dowling et W. McKinnon. Cambridge University Press, 59-78.
} 